\title{
MicroRNA-20a-5p targets RUNX3 to regulate proliferation and migration of human hepatocellular cancer cells
}

\author{
YANKE CHEN $^{1,2^{*}}$, XIAOFEI WANG ${ }^{2 *}$, JIWEN CHENG $^{4}$, ZHEN WANG $^{2}$, TING JIANG ${ }^{2}$, \\ NI HOU ${ }^{2}, \mathrm{NALIU}^{2}$, TUSHENG SONG ${ }^{2}$ and CHEN HUANG ${ }^{1-3}$ \\ ${ }^{1}$ Experiment Center of Biomedical Research, ${ }^{2}$ Department of Genetics and Cell Biology, and ${ }^{3}$ Key Laboratory of \\ Environmentally and Genetically Associated Diseases, Xi'an Jiaotong University School of Medicine, Xi'an, \\ Shaanxi 710061; ${ }^{4}$ Departments of Hepatobiliary Surgery of The First Affiliated Hospital, \\ Xi'an Jiaotong University School of Medicine, Health Science Center, \\ Xi'an, Shaanxi 710061, P.R. China
}

Received March 18,2016; Accepted August 17, 2016

DOI: 10.3892/or.2016.5144

\begin{abstract}
Growing evidence indicates that some abnormally expressed microRNAs (miRNAs) influence tumorigenesis and progression. Previous studies reported that miR-20a is among the frequently altered miRNAs in human hepatocellular carcinoma (HCC), but its expression pattern and role in HCC remain controversial. In the present study, we demonstrated that miR-20a-5p exhibited aberrant expression in HCC tissues compared with paired non-tumor tissues: $52 \%$ of the tumor samples showed a greater increase. Overexpression of miR-20a contributed to HCC cell proliferation and migration in vitro, and treatment with anti-miR20a-5p caused the opposite effects. Further studies revealed RUNX3, an important tumor-suppressor, as a direct target of miR-20a-5p. We observed that the level of RUNX3 was sharply reduced in both mRNA and protein in HCC tissues compared with paired nontumor tissues. Collectively, our results support the viewpoint that miR-20-5p has an oncogenic property, miR-20a overexpression contributed to HCC cell proliferation and migration through reducing the translation of RUNX3. The data provide a new mechanism of miR-20a regulating RUNX3 in HCC.
\end{abstract}

\section{Introduction}

MicroRNAs (miRNAs) are endogenous small RNA molecules that regulate gene expression by binding to specific messenger

Correspondence to: Professor Chen Huang or Professor Tusheng Song, Department of Genetics and Molecular Biology, Xi'an Jiaotong University School of Medicine, 76 Yanta Western Road, Xi'an, Shaanxi 710061, P.R. China

E-mail: hchen@mail.xjtu.edu.cn

E-mail: tusheng@mail.xjtu.edu.cn

${ }^{*}$ Contributed equally

Key words: miR-20a, human hepatocellular cancer, RUNX3
RNAs (mRNAs). miRNA genes are expressed as primary transcripts (pri-miRNAs) in the nucleus and further processed into stem-loop precursor miRNAs (pre-miRNAs) by the action of enzymes Drosha RNase III endonuclease $(1,2)$. The miRNA precursor is then exported to the cytoplasm by means of the nuclear export receptor, exportin-5. Once it is in the cytoplasm, pre-miRNAs are cleaved by Dicer and TRBP, resulting in the production of 22 -nucleotide double-chain mature miRNAs (2). The mature miRNA subsequently was loaded onto an AGO protein to form an effecter complex called RNA-induced silencing complex (RISC), which binds to the 3'-untranslated regions (3'UTRs) of their target mRNAs through imperfect base pairing, and mediates either endonucleolytic cleavage or translational repression of target mRNAs (3-6). Since various abnormally expressed miRNAs were found to influence tumorigenesis and progression by regulating oncogenes and tumor-suppressor genes, researchers have tried to identify specific miRNAs for the diagnosis and treatment of tumors (7-9).

Hepatocellular carcinoma (HCC) is one of the most fatal cancers in Asia and Africa. Previous studies have shown that miRNA expression profiles are significantly changed in human HCC (10-12). Until now several miRNAs have been discovered associated with poor prognosis and identified as candidate biomarkers in human HCC, such as miR-26a (13), miR-30d (14), miR-122 (15,16), and miR-17 family (17). The miR-17 family is composed of the highly conserved miR106b-25/miR-17-92 cluster. This miRNA cluster is reported to be overexpressed in HCC clinical samples (17). miR-20a belongs to the miR-17-92 cluster and is located on chromosome 13. Although the aberrant expression of miR-20a in HCC has been observed, data on its function is inconsistent. Li et al (17) examined the expression of miR-17 family in 56 pairs of HCC samples and the corresponding paired non-tumor liver using reverse transcription-real-time PCR (qRT-PCR). They reported at least 52\% of the HCC samples showed a greater than two-fold increase in the expression for miR-20a; and miR-17 family (including miR-20a) was necessary for cell proliferation and anchorage-independent growth. Thus, they considered miR-20a had oncogenic potential. 
However, Fan et al (18) reported that miR-20a was significantly decreased in HCC samples compared with normal liver tissue. In addition, patients with lower miR-20a expression had significantly poorer recurrence-free and overall survival. Restoration of miR-20a suppressed hepatoma cell proliferation and induced G1 arrest in cell cycle and apoptosis by directly targeted myeloid cell leukemia sequence 1 (Mcl-1), so it may be a tumor-suppressor in HCC. Thus, the expression and function of miR-20a in HCC have not been fully elucidated, and need to be clarified.

The information from exploratory analysis using bioinformatics algorithms (TargetScan, Mirnaviewer and DIANA-MicroTest) indicated that the 3'UTR of RUNX3 harbored a potential binding site of miR-20a-5p, which suggested that miR-20a-5p plays a role in regulating RUNX3 expression. The human RUNX3 belongs to the RUNX gene family (the runt-related transcription factor). Clinical and experimental data have shown that functional inactivation of RUNX3 is frequently observed in multiple solid tumors and highly associated with tumor progression, lymph node metastasis and poor prognosis (19-23). Thus, it has been regarded as a tumor-suppressor. Previous studies have attributed the loss or reduction of RUNX3 expression to hemizygous deletion (24-26) and epigenetic silencing (27,28). To date, several miRNAs were identified as regulators of RUNX3 in carcinomas, such as miR-532-5p in cutaneous melanoma (29), and miR130b (30) and miR30a (31) in gastric cancer. Nonetheless, the mechanism underlying the regulation of RUNX3 expression through miRNAs remains poorly explored.

The aims of the present study were: i) to clarify the expression and function of miR-20a-5p in HCC; and ii) to determine whether RUNX3 is a target of miR-20a-5p and evaluate the role of this mechanism in HCC. Previously, Li et al have reported the precursors and the mature miRNAs of the miR-106b-25 cluster were highly expressed in HCC tissues and HepG2, and Huh7 hepatoma-derived cells, while only low levels of expression were observed in primary hepatocytes (17). In another study, Nakanishi et al, reported that RUNX3 mRNA and protein were undetectable in eight hepatoma-derived cell lines (HepG2, Hep3B, Huh1, Huh7, JHH1, JHH2, JHH4 and HLE), in HLF and SK-Hep1 cells, RUNX3 mRNA and protein were significantly under-expressed (32). Our experimental results showed that miR-20a was highly expressed and RUNX3 was lower expressed in SMMC-7721 hepatoma cell, thus, we used SMMC-7721 cells in the present study to clarify the function of miR-20a and to verify the hypothesis of RUNX3 as a target of miR-20a-5p in HCC.

\section{Materials and methods}

Cell lines. Human hepatoma SMMC-7721 and human embryonic kidney HEK293T cells were provided by the Institute of Cell and Biochemistry, Chinese Academy of Sciences (Shanghai, China) and cultured in RPMI-1640 medium supplemented with 10\% fetal bovine serum (FBS) (both from PAA Laboratories, Pasching, Austria) at $37^{\circ} \mathrm{C}$ under a mixture of $95 \%$ air and $5 \% \mathrm{CO}_{2}$.

Clinical specimens. Formalin-fixed paraffin-embedded (FFPE) tissue blocks of tumor and corresponding adjacent non-tumorous liver tissues from 39 cases of HCC patients were obtained from the Department of Pathology, and 14 cases of fresh frozen clinical specimens were collected from HCC patients who underwent hepatectomy in the Department of Surgery of The First Affiliated Hospital of Xi'an Jiaotong University. No patients received local or systemic therapies before surgery and both tumor and matched adjacent non-tumor tissue were histologically confirmed. The present study was conducted under a protocol approved by the Hospital Ethics Committee and informed consent was obtained from each patient.

Reverse transcription quantitative real-time polymerase chain reaction ( $q R T-P C R)$. Total RNA from cells and frozen tissues was extracted with TRIzol reagent (Invitrogen, Carlsbad, CA, USA), and from FFPE clinical samples with RecoverAll ${ }^{\mathrm{TM}}$ Total Nucleic Acid Isolation kit (Ambion, Austin, TX, USA) following the manufacturer's protocol. For detection the expression level of miR-20a-5p and RUNX3, 500 ng RNA was reverse transcribed using PrimeScript RT Master Mix (Takara, Otsu, Japan). The primers are listed in Table I. Subsequently the cDNA was amplified by SYBR Premix Ex $T a q^{\mathrm{TM}}$ II (Takara). U6 snRNA and GAPDH were used as controls for miRNA and mRNA level, respectively. Relative quantitation was calculated using the $2^{-\Delta \Delta \mathrm{Ct}}$ method. All experiments were performed in triplicates.

Immunohistochemistry. For immunohistochemical detection of RUNX3 expression in tumor and corresponding non-tumorous tissues from HCC patients, these tissues were cut at the thickness of $5-\mu \mathrm{m}$. The sections were collected and processed for RUNX3 immunohistochemistry with the avidin-biotin peroxidase complex (ABC) method following the manufacturer's protocol.

Construction of human miR-20a precursor expression vector. Human miR-20a precursor (pre-miR-20) was synthesized by chemical method by Shanghai Sangon Biological Engineering Technology and Services Co. Ltd. (Shanghai, China). Then, pre-miR-20 was subcloned between the EcoRI and HindIII sites of the pcDNA6.2-GW/EmGFP vector (Invitrogen). The sequences of constructed plasmids were confirmed by DNA sequencing (Sangon Biotech, Shanghai, China).

Transfection of miRNA or inhibitor into SMMC-7721 cells. Anti-miR-20a-5p inhibitor and scrambled control fragment were synthesized by GenePharma Company (Shanghai, China). The sequence information of anti-miR-20a-5p inhibitor is presented in Table I. SMMC-7721 cells at $70-80 \%$ confluency, were transfected with pre-miR-20a-pcDNA6.2-GW $(0.2-2 \mu \mathrm{g})$ or anti-miR-20a-5p inhibitor (16-160 pmol) using Lipofectamine 2000 (Invitrogen) method following the manufacturer's protocol. The control vector pcDNA6.2-GW $(0.2-2 \mu \mathrm{g})$ or scrambled control RNA (16-160 pmol) were also transfected as negative controls.

Cell proliferation assay by Cell Counting Kit-8 (CCK-8) assay. SMMC-7721 cell proliferation was measured by CCK- 8 assay. Briefly, cells were seeded into 96 -well culture plates at a density of $6 \times 10^{3}$ cells/well. Transfection was performed the next day at the concentration described above. The in vitro proliferation 
Table I. Sequences of primers and inhibitor used in the study.

\begin{tabular}{ll}
\hline Name & \multicolumn{1}{c}{ Sequences } \\
\hline miR-20a-RT & GTCGTATCCAGTGCGTGTCGTGGAG \\
& TCGGCAATTGCACTGGATACGACCT \\
& ACCTG \\
miR-20a-5p-F & ATCCAGTGCGTGTCGTG \\
miR-20a-5p-R & TGCTTAAAGTGCTTATAGTG \\
U6-RT & CGCTTCACGAATTTGCGTGTCAT \\
U6-F & GCTTCGGCAGCACATATACTAAAAT \\
U6-R & CGCTTCACGAATTTGCGTGTCAT \\
Anti-miR-20a-5p & 5'-CTACCTGCACTATAAGCACTTTA-3' \\
Scrambled & 5'-TGACTGTACTGAACTCGACTG-3' \\
\hline
\end{tabular}

ability of SMMC-7721 cells were measured over 24 and $48 \mathrm{~h}$ using the CCK-8 kit (Boster, Wuhan, China) assay according to the manufacturer's instructions. Absorbance was measured at a wavelength of $450 \mathrm{~nm}$ in a microplate spectrophotometer.

Cell migration assay by wound-healing test. SMMC-7721 cell migration ability was measured by a wound-healing assay. Full confluent cells were seeded into 24 -well plates. A cellular area was created by scraping using a pipette tip. Wound closure was measured at 12, 24, 36 and $48 \mathrm{~h}$ intervals.

Western blot analysis. Cells were lysed in RIPA buffer (Beyotime Biotech, Jiangsu, China) and protein concentrations were measured by the BCA assay (Pierce Biotechnology, Inc., Rockford, IL, USA). Total protein (10 $\mu \mathrm{g})$ was separated on a $12 \%$ SDS-PAGE and transferred onto polyvinylidene difluoride (PVDF). membranes. The PVDF membranes were subsequently immune-blotted with the appropriate primary antibodies including mouse anti-human RUNX3 monoclonal antibody (Abcam), or mouse anti-human actin monoclonal antibody (Santa Cruz Biotechnology, Santa Cruz, CA, USA). After extensive washing, the membranes were incubated with a horseradish peroxidase-conjugated goat anti-mouse secondary antibody (Pierce Biotechnology, Inc.). Signals were detected by an ECL kit (Pierce Biotechnology, Inc.) according to the manufacturer's instructions.

Luciferase reporter assay. The 3'UTR of RUNX3 (NM-001031680) containing the predicted miR-20a-5p binding site was constructed. The mutant RUNX3 3'UTR was created by mutating multiple nucleotides complementary to the miR20a-5p seed region. The sequences of constructed plasmids were confirmed by DNA sequencing (Sangon Biotech).

HEK293T cells were cultured into 96-well plates with $50-70 \%$ confluency at $24 \mathrm{~h}$ before transfection. A mixture of $100 \mathrm{ng}$ pmiR-RB-Report ${ }^{\mathrm{TM}}$ h-RUNX3 wild-type (WT) or mutant (Mut) reporter plasmid vector along with $50 \mathrm{nM}$ pre-miR-20a-pcDNA6.2-GW or corresponding control vectors pcDNA6.2-GW were co-transfected. The luciferase activity was measured $48 \mathrm{~h}$ post-transfection using Dual-Glo Luciferase Assay System (Promega, Madison, WI, USA) with Renilla (Rluc) luciferase activity as the reporter gene and

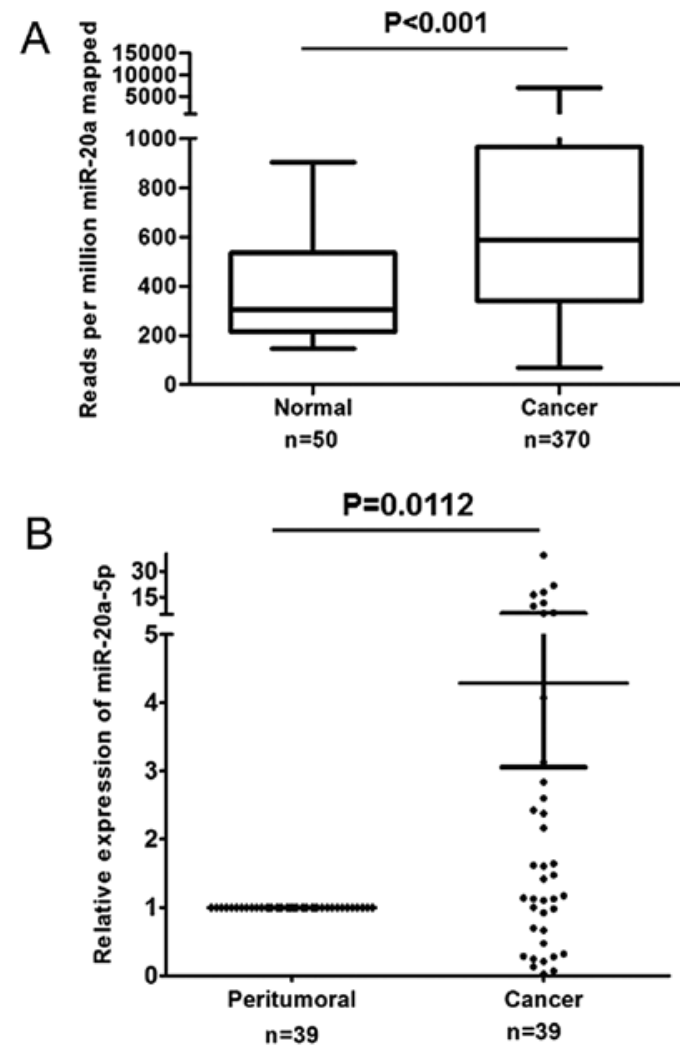

Figure 1. The expression of miR-20a is aberrant in tumor tissues of HCC, compared with normal liver tissue or the corresponding paired non-tumor tissues. (A) The data from The Cancer Genome Atlas (TCGA) database showing increased expression of miRNA-20a was found in HCC tumor samples $(n=370)$ compared with normal liver tissue $(n=50)$. The levels of miRNA-20a expression were assayed by deep sequencing. (B) The data from qRT-PCR analysis showed an overview of the miR-20a-5p dysregulated in HCC tumor samples, compared with the corresponding paired non-tumor tissues $(n=39)$. The relative expression level of miR-20a-5p was normalized to the mean of U6 RNA. Data were from three independent experiments and represented as mean $\pm \mathrm{SEM} ; \mathrm{P}<0.05$.

firefly luciferase (Luc) as the reference gene. Each assay was repeated in triplicate.

Statistical analysis. Statistical evaluation was performed using SPSS software (version 11.05; SPSS, Inc., Chicago, IL, USA). The statistical analysis between the two groups was conducted with Student's t-test. Correlations between miR-20a-5p clinicopathological features were analyzed using Kaplan-Meier and univariate Cox proportional hazard regression. Moreover, multiple comparisons between the groups were performed using $\mathrm{S}-\mathrm{N}-\mathrm{K}$ method. $\mathrm{P}<0.05$ was considered to indicate a statistically significant result.

\section{Results}

miR-20a-5p expression is aberrant in HCCs. miR-20a is among the most frequently altered miRNAs in human cancers. To investigate the expression of miR-20a in liver cancer, we collected the information of miR-20a expression in the large cohorts of HCC patients available from The Cancer Genome Atlas (TCGA) database (Liver hepatocellular carcinoma: BCGSC IlluminaHiSeq miRNASeq). The data are presented in Fig. 1A, miR-20a is significantly increased in HCC tissues 
A
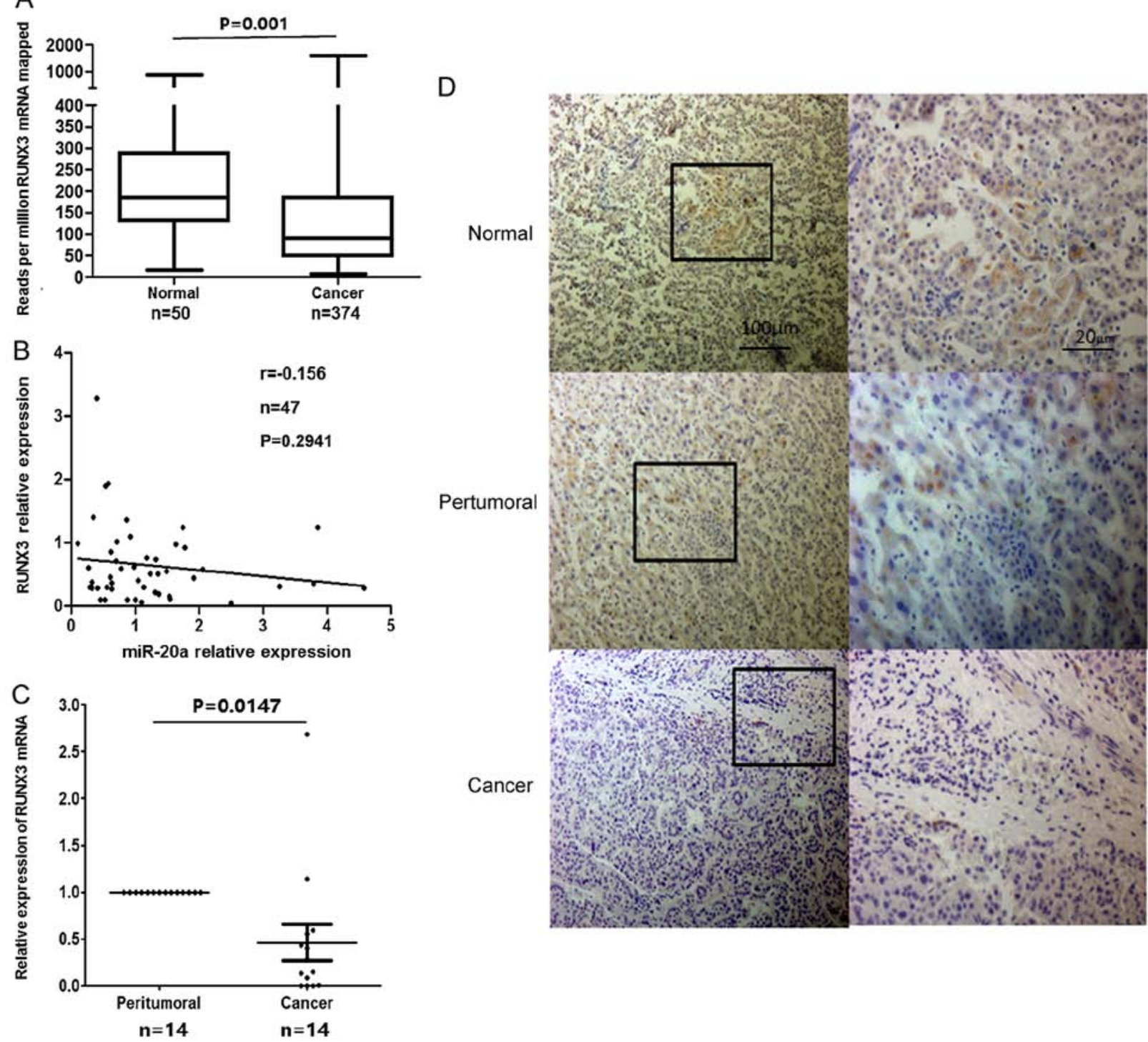

Cancer

Figure 2. RUNX3 is strikingly downregulated in tumor tissues of HCC, compared with normal liver tissue or the corresponding paired non-tumor tissues. (A) The data from TCGA database showing down-expression of RUNX3 was found in HCC tumor samples (n=374) compared with normal liver tissue (n=50). The levels of RUNX3 mRNA were assayed by deep sequencing. (B) The correlation between expression RUNX3 and miR-20a in HCC tumor samples and paired non-tumor tissues $(n=47)$ was analyzed using the information from TCGA database. (C) The relative expression level of RUNX3 mRNA was evaluated in 14 cases of frozen HCC specimens by qRT-PCR. All RUNX3 expression levels were normalized to the mean of GAPDH mRNA. Data are from three independent experiments and presented as mean \pm SEM. (D) Representative images of RUNX3 protein expression were observed by immunohistochemistry staining.

compared with normal liver tissues $(\mathrm{P}<0.001)$. To validate the expression of miR-20a in liver cancer, we evaluated the expression of miR-20a-5p patterns in 39 pairs of HCC and adjacent non-tumor clinical tissue specimen by qRT-PCR. As shown in Fig. 1B, an aberrant expression phenomenon of miR-20a-5p was observed in HCC tissues compared with the corresponding paired non-tumor tissues. Over $52 \%$ of the samples showed a $>1.2$-fold upregulation for all members of miR-20a-5p; $28 \%$ of the samples showed a $>1.2$-fold downregulation; and $20 \%$ of the samples showed no significant changes. These results showed that miR-20a-5p expression was aberrant in human primary HCCs. The relationships between miR-20a-5p expression and clinicopathological features were analyzed using Kaplan-Meier and univariate Cox proportional hazard regression; the data showed that miR-20a-5p expres- sion had no association with clinicopathological information (data not shown).

RUNX3 is downregulated in HCC. There are abundant data reported that the expression of RUNX3 was poor in tumor tissues. We analyzed variation of RUNX3 in HCC using the data from TCGA database (Liver hepatocellular carcinoma: UNC IlluminaHiSeq RNASeq, UNC IlluminaHiSeq RNASeqV2). The information showed that RUNX3 is significantly decreased in $\mathrm{HCC}$ tissues compared with no-corresponding normal liver tissues $(\mathrm{P}=0.001)$ (Fig. 2A). Furthermore, the RUNX3 protein was predicted as a candidate target of miR-20a-5p using bioinformatics analysis. We assayed the correlations between miR-20a levels and RUNX3 protein. The information was from 47 pairs of corresponding data in 

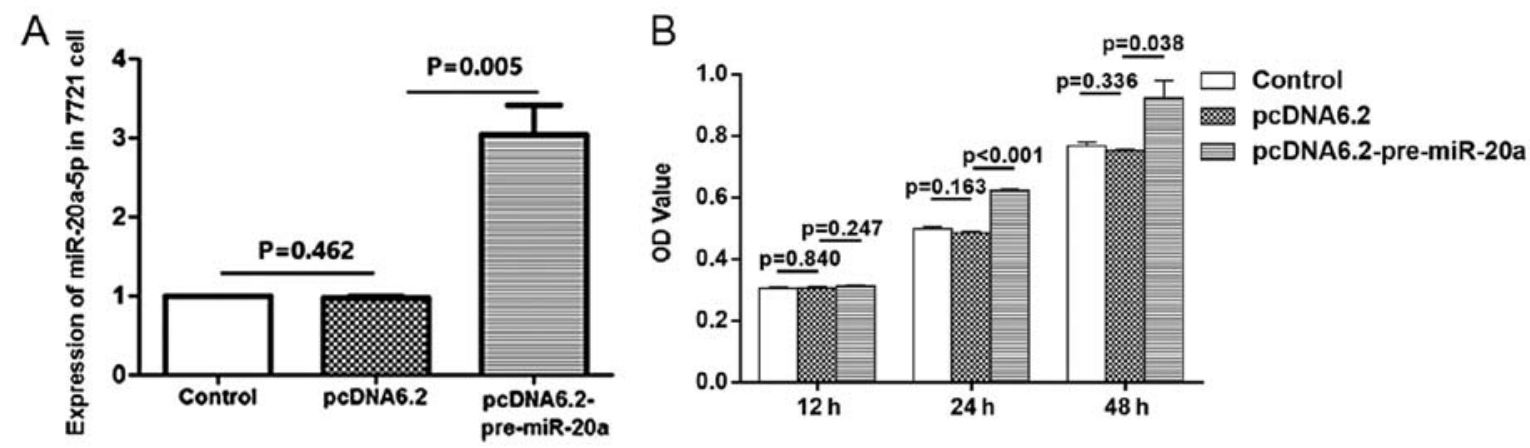

C
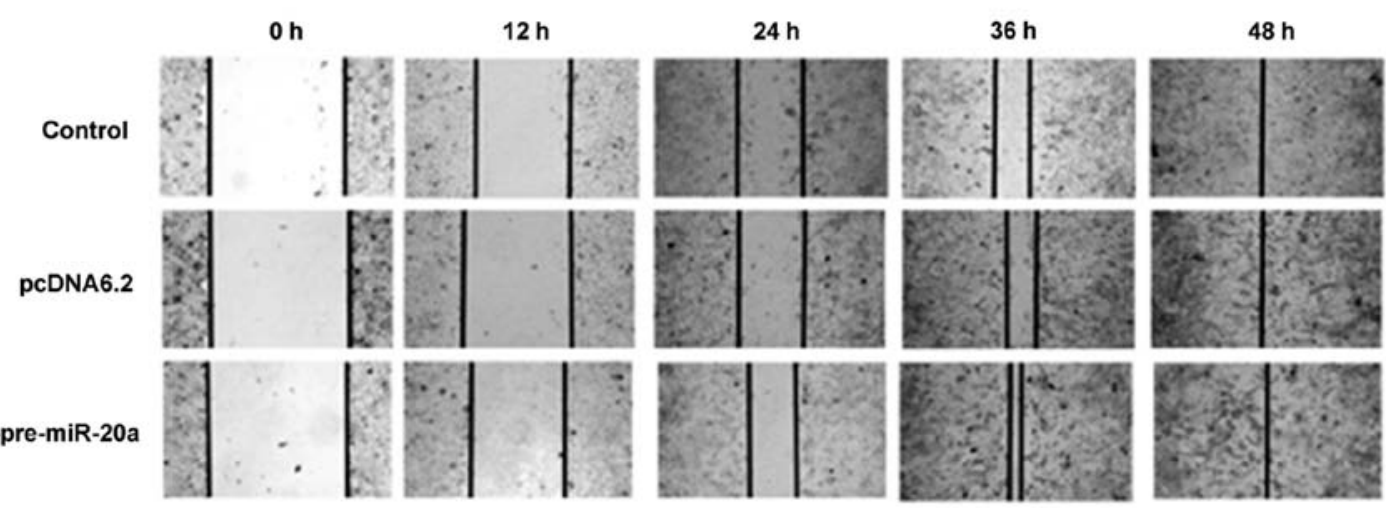

Figure 3. Overexpression of miR-20a in SMMC-7721 cells enhances cell proliferation and migration. (A) The relative expression level of miR-20a-5p was examined by qRT-PCR in SMMC-7721 cells transfected with pcDNA6.2 or pcDNA6.2-miR-20a. (B) At 24 and $48 \mathrm{~h}$ after transfection with pcDNA6.2 or pcDNA6.2-miR20a, cell proliferation was examined by the CCK-8 assay. (C) The effect of transfection of pcDNA6.2 or pcDNA6.2-miR20a on the cell migration was observed by wound-healing test.

TCGA datasets. The results showed that the expression level of miR-20a and RUNX3 mRNA presented a trend of negative correlation (Fig. 2B; $r=-0.156, P=0.2941$ ).

Next, we examined the mRNA and protein expression of RUNX3 in clinical specimens of HCC patients using qRT-PCR and IHC analysis. qRT-PCR analysis from 14 paired specimens showed that most HCC patients $(12 / 14)$ exhibited significantly decreased level of RUNX3 mRNA in hepatoma tissues compared with the paired non-tumor tissues (Fig. 2C). Consistently, IHC staining showed that RUNX3 was expressed in non-tumor tissue and mainly located in the cytoplasm and nucleus, but it was very rare in hepatoma tissues (Fig. 2D).

Overexpression of $\mathrm{miR}-20$ a promotes the proliferation and migration of SMMC-7721 hepatoma cancer cells. To investigate the effect of miR-20a on cell proliferation and migration, we performed both overexpression studies using plasmid of miR-20a precursor and inhibition studies using miR-20a-5pspecific antisense oligonucleotide inhibitor (anti-miR-20a-5p). First, we transfected $2 \mu \mathrm{g}$ of plasmid into SMMC-7721 cells. The efficiency of transfection was confirmed by qRT-PCR. At $48 \mathrm{~h}$ post-transfection with miR-20a precursor plasmid (pre-miR-20a-pcDNA6.2-GW), the expression of miR-20a-5p was increased $\sim 3$-fold (Fig. 3A). The data from CCK-8 assay showed an increase in proliferation occurred in cells after transfection with miR-20a precursors plasmid (Fig. 3B). The wound healing assay showed that miR-20a overexpression also enhanced cell migration ability in vitro (Fig. 3C). Accordingly, treatment with anti-miR20a-5p inhibitor caused the opposite effects. Compared with the cells which were treated with scrambled control oligonucleotides, anti-miR-20a-5p inhibitor decreased cell proliferation and migration at all-time points studied (Fig. 4).

When performing analysis on cell migration, and proliferation behavior of cells affecting migration, in the present study, we overexpressed or inhibited miR-20a in SMMC-7721 cells, and we observed the change of proliferation and migration from 12 to $48 \mathrm{~h}$. The data showed an increase in proliferation occurred when cells were transfected with pre-miR-20a after $24 \mathrm{~h}$, at the time of $12 \mathrm{~h}$ cell proliferation did not significantly change. At the same time $(12 \mathrm{~h})$, the migration of cells was influenced significantly by miR-20a. Therefore, we believe that cell migration detected by wound healing assay was not due to the cell proliferation at this time point. These data indicated that HCC cell growth and migration could be modulated by expression of miR-20a-5p.

miR-20a-5p directly targets the RUNX3 expression. Further analysis was carried out to determine whether miR-20a-5p regulated the expression of RUNX3. The result from western blotting showed overexpression of miR-20a led to decreased level of RUNX3 protein (Fig. 5A). Accordingly, transfection of the anti-miR-20a-5p inhibitor recovered the level of RUNX3 protein (Fig. 5B). However, treatment of SMMC-7721 cells with miR-20a precursor plasmid or anti-miR-20a-5p inhibitors for $48 \mathrm{~h}$ did not affect the level of RUNX3 mRNA as determined by qRT-PCR (Fig. 5C and D). The data suggested that miR-20a-5p reduced RUNX3 expression during protein translation. 

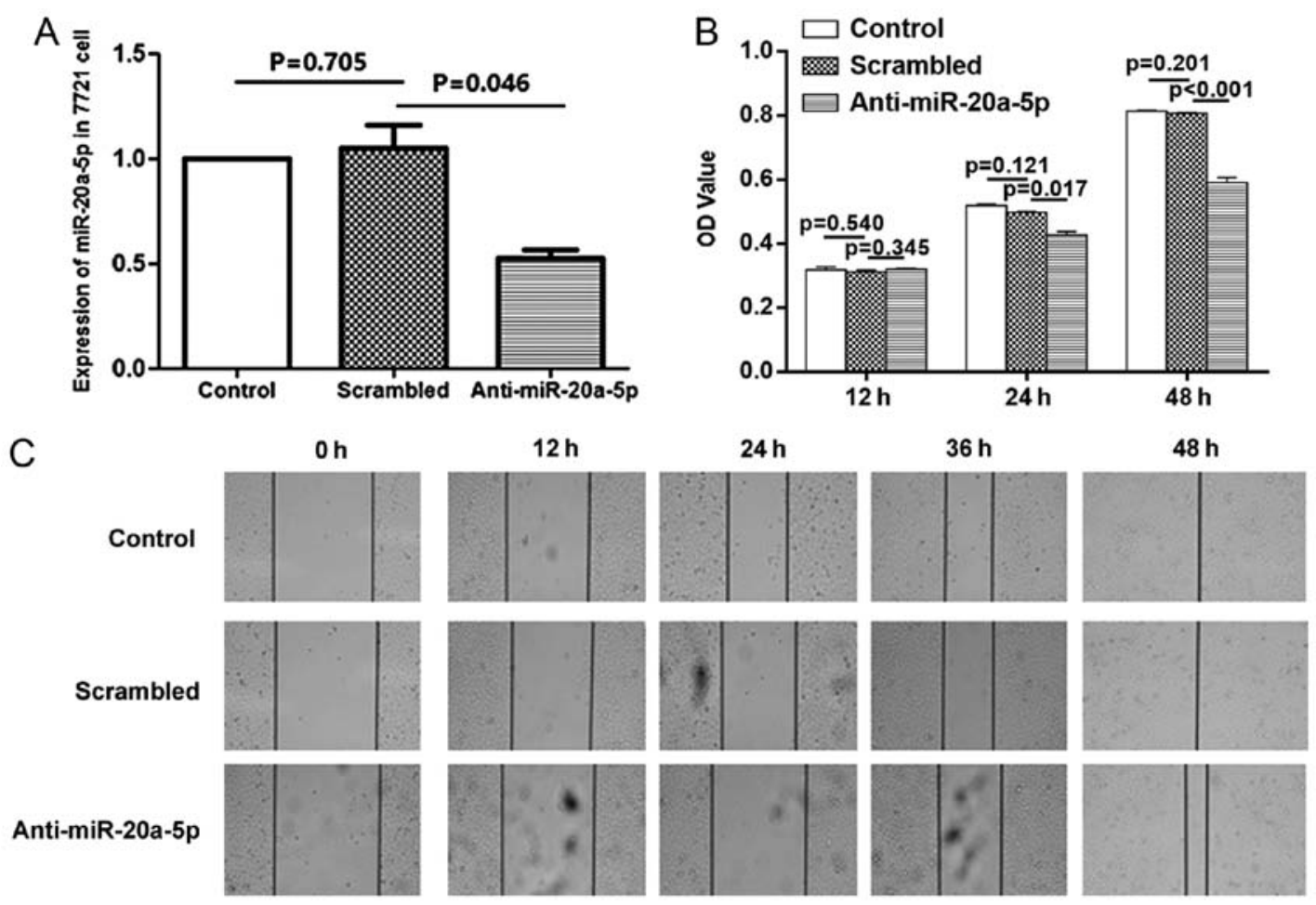

Figure 4. Neutralization of endogenous miRNA-20a-5p by miRNA inhibitor reduces SMMC-7721 cell proliferation and migration. (A) The relative expression level of miR-20a-5p was examined by qRT-PCR in SMMC-7721 cells treated with scrambled or anti-miR-20a-5p inhibitor. (B) At 24 and $48 \mathrm{~h}$ after transfection with scrambled or anti-miR-20a-5p inhibitor, cell proliferation was examined by the CCK-8 assay. (C) The effect of transfection of scrambled or anti-miR-20a-5p inhibitor on the cell migration was observed by wound-healing test.

To assess whether miR-20a-5p directly alters the expression of RUNX3, a fragment of the 3'UTR of RUNX3 mRNA containing the putative miR-20a-5p-binding sequence, cloned into the pmiRGLO dual-luciferase reporter vector (RUNX3-WT), and a mutations were generated in the RUNX3 sequences by mutating $4 \mathrm{nt}$ for the seed region (RUNX3-MT), as indicated in Fig. 5E. Then, HEK293 cells were co-transfected with miR-20a and RUNX3-WT or RUNX3-MT 3'UTR vector. It was observed that in the presence of miR-20a-5p, these relative luciferase activities were significantly reduced ( $\mathrm{P}=0.013)$, but miR-20a-5p failed to inhibit the luciferase activity of the RUNX3-MT reporter vector (Fig. 5F), which indicated that miR-20a-5p modulated gene expression directly at the 3 'UTR of RUNX3. Thus, these data provide evidence that miR-20a-5p interacts with the 3'UTR of the RUNX3 transcript and regulating its translation.

\section{Discussion}

miR-20a is among the frequently altered microRNAs (miRNAs) in HCC, but its expression pattern and role in HCC still remain controversial. In the present study, we examined miR-20a expression levels in 39 ormalin-fixed paraffinembedded (FFPE) and 14 frozen tissues of patients with HCC. Compared with the corresponding paired non-tumor samples, miR-20a expression was aberrant, consistent with the observation from Li et al (17); $52 \%$ of the tumor samples showed a greater increase in the expression for miR-20a in tumor tissue. Similar results have been described in other members of miR-17 family in miRNA profiling analysis in $\operatorname{HCC}(10,11,17,33,34)$, although other studies did not observe these changes $(12,35)$, even opposite results (18). The difference could be due to the differences in profiling techniques and control group used. In the studies of Fan et al and Jiang et al, the change of miR-20a in HCC tumor tissue compared with the no-paired normal liver specimens was evaluated. In contrast, in the study of Li et al and ours HCC with their corresponding paired non-tumor specimens were compared. Previously, Li et al reported that the precursors and the mature miRNAs of the miR-17 family were highly expressed in HCC (17), and we also examined the expression of the other members, and our result was conformity with the observation from Li et al (17). Theoretically, the members should have a similar effect, but differences cannot be ruled out. Therefore, we may pay attention to these differences in the follow-up study.

Furthermore, the RUNX3 protein, a tumor-suppressor, was predicted as a candidate target of miR-20a-5p by bioinformatics analysis. This assay along with the low expression of RUNX3 in HCC indicates that RUNX3 may be downregulated by miR-20a-5p. Indeed, we observed that transfection with miR-20a suppressed RUNX3 protein expression and enhanced cell proliferation and migration in HCC cells. Treatment with anti-miR20a-5p caused the opposite effects. Data from luciferase reporter assay further evidenced RUNX3 was a direct target of miR-20a-5p.

RUNX3 as a transcription factor, participates in the aetiology of diverse cancers, and is consider to have a role in tumor suppression since it is either not expressed or has undefined expression in the nucleus of many cancers. Nonetheless, in some cancers, there is evidence that the RUNX3 is expressed 
A

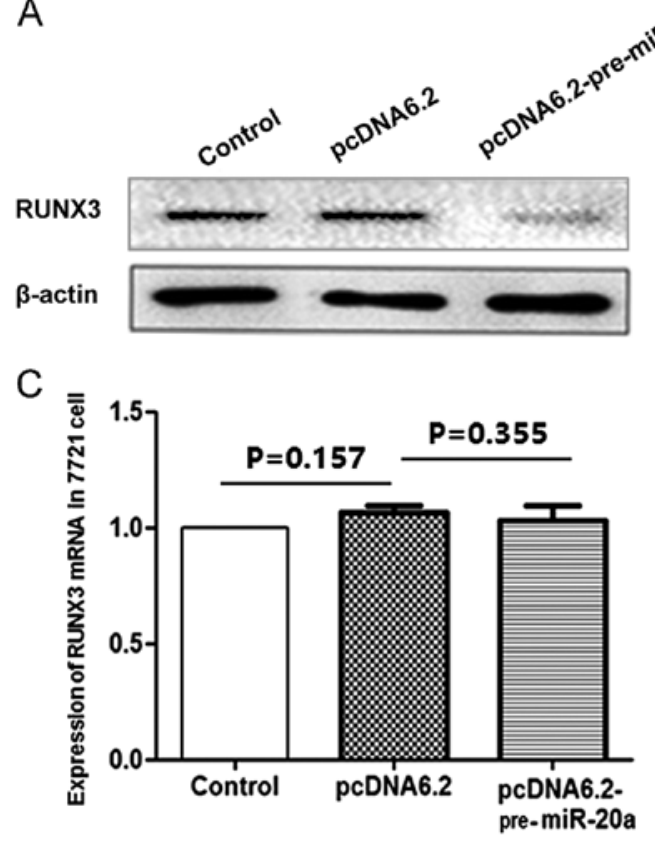

E

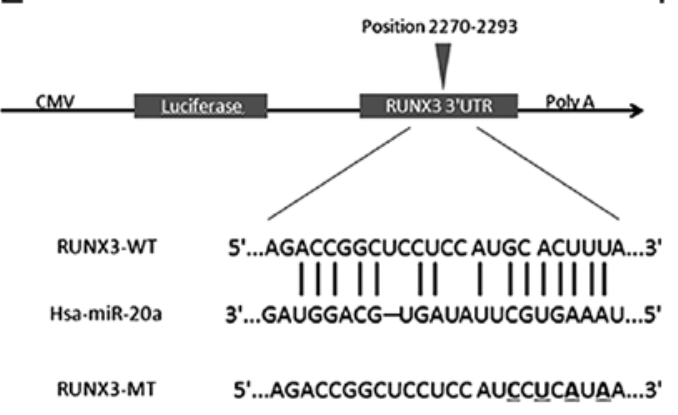

B control scrambled Antim-mir-202.5p

RUNX3

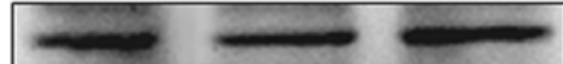

$\beta$-actin

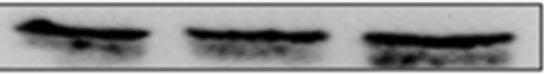

D

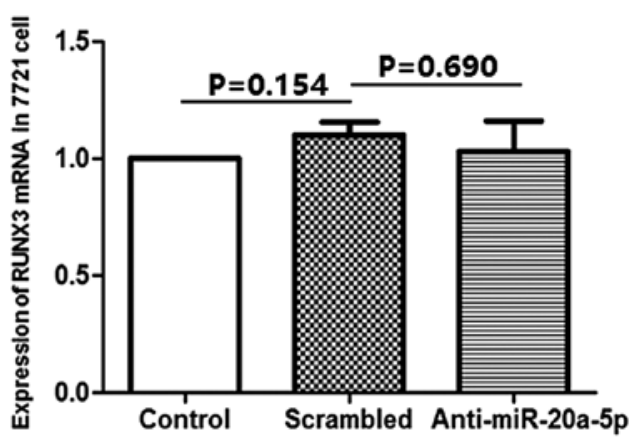

$\mathrm{F}$

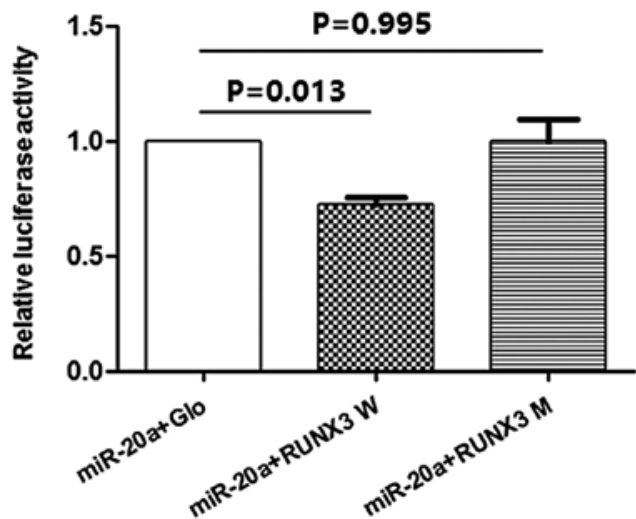

Figure 5. miR-20a-5p downregulates the protein level of RUNX3 by targeting its 3'UTR. (A) The expression level of RUNX3 were measured after transfection of miR-20 expression vector or anti-miR-20a-5p inhibitor by (A and B) western blotting and (C and D) qRT-PCR. (E) Putative miR-20a-5p-binding sites in the RUNX3 3'UTRs, mutations were generated in the RUNX3 sequences by mutating 4 nt in the seed region of miR-20a-5p as indicated. (F) The wild-type or mutant 3'UTRs (indicated as WT or MT) of RUNX3 were cloned into the downstream of the luciferase reporter vector and then co-transfected into HEK293 cells with pre-miR-20a. The relative luciferase activities were measured $48 \mathrm{~h}$ post transfection and normalized by calculating the ratio of firefly luciferase with Renilla luciferase activity.

in the cytoplasm (36). In the present study, using immunohistochemistry we found that the RUNX3 protein was slightly expressed in HCC with both cytoplasmic and nuclear patterns. This protein mislocalization has also been found in various types of human cancers $(37,38)$. Various researchers believe that, when RUNX3 is expressed in the cytoplasm, it is thought to be in its inactive state, but this hypothesis needs to be confirmed by experiments.

Previous studies have demonstrated that losing RUNX3 contributed to tumor progress. RUNX3 suppressed the cancer cell growth through regulating the expression of Akt and cyclin D1 (38), and the pathways of TGF- $\beta$, Wnt and Notch signaling (39). Loss of RUNX3 expression was highly associated with increased lymph node metastasis, reduced cellular differentiation and shorter survival duration in gastric cancer patients (21). In addition, RUNX3 also slowed down cancer metastasis through inhibition of epithelial-mesenchymal transition (EMT) (40). Since these studies have already demonstrated that RUNX3 is a tumor-suppressor and inhibit cell proliferation and migration, here we just postulate that miR-20a-5p influence cell proliferation and migration through regulating RUNX3 expression, although we cannot exclude the possibility that other targets may contribute mediating the action of miR-20a-5p, as various studies have demonstrated that miR-20a regulates the important tumor suppressor, PTEN, in $\mathrm{HCC}$ (41).

Taken together, our results support the viewpoint that miR20-5p has an oncogenic property in HCC; it enhances cancer cell proliferation and migration through reducing the translation of RUNX3.

\section{Acknowledgements}

The present study was supported by Grants from the National Natural Science Foundation of China (no. 81101744), the Fundamental Research Funds for the Central Universities (no. xjj2014144), and the Funds for Technical Resources sharing Platform of Shaanxi Province, China (No. 2015FWPT-14). 


\section{References}

1. Lee Y, Jeon K, Lee JT, Kim S and Kim VN: MicroRNA maturation: Stepwise processing and subcellular localization. EMBO J 21: 4663-4670, 2002.

2. Lee Y, Ahn C, Han J, Choi H, Kim J, Yim J, Lee J, Provost P, Rådmark O, Kim S, et al: The nuclear RNase III Drosha initiates microRNA processing. Nature 425: 415-419, 2003.

3. Bartel B and Bartel DP: MicroRNAs: At the root of plant development? Plant Physiol 132: 709-717, 2003.

4. Khvorova A, Reynolds A and Jayasena SD: Functional siRNAs and miRNAs exhibit strand bias. Cell 115: 209-216, 2003.

5. Doench JG, Petersen CP and Sharp PA: siRNAs can function as miRNAs. Genes Dev 17: 438-442, 2003.

6. Carrington JC and Ambros V: Role of microRNAs in plant and animal development. Science 301: 336-338, 2003.

7. Calin GA and Croce CM: MicroRNA signatures in human cancers. Nat Rev Cancer 6: 857-866, 2006.

8. Lu J, Getz G, Miska EA, Alvarez-Saavedra E, Lamb J, Peck D, Sweet-Cordero A, Ebert BL, Mak RH, Ferrando AA, et al: MicroRNA expression profiles classify human cancers. Nature 435: 834-838, 2005

9. Lujambio A and Lowe SW: The microcosmos of cancer. Nature 482: 347-355, 2012.

10. Murakami Y, Yasuda T, Saigo K, Urashima T, Toyoda H, Okanoue $\mathrm{T}$ and Shimotohno K: Comprehensive analysis of microRNA expression patterns in hepatocellular carcinoma and non-tumorous tissues. Oncogene 25: 2537-2545, 2006.

11. Huang YS, Dai Y, Yu XF, Bao SY, Yin YB, Tang M and Hu CX: Microarray analysis of microRNA expression in hepatocellular carcinoma and non-tumorous tissues without viral hepatitis. J Gastroenterol Hepatol 23: 87-94, 2008.

12. Jiang J, Gusev Y, Aderca I, Mettler TA, Nagorney DM, Brackett DJ, Roberts LR and Schmittgen TD: Association of MicroRNA expression in hepatocellular carcinomas with hepatitis infection, cirrhosis, and patient survival. Clin Cancer Res 14: 419-427, 2008.

13. Yang X, Liang L, Zhang XF, Jia HL, Qin Y, Zhu XC, Gao XM, Qiao P, Zheng Y, Sheng YY, et al: MicroRNA-26a suppresses tumor growth and metastasis of human hepatocellular carcinoma by targeting IL-6-Stat3 pathway. Hepatology 58: 158-170, 2013.

14. Yao J, Liang L, Huang S, Ding J, Tan N, Zhao Y, Yan M, Ge C, Zhang Z, Chen T, et al: MicroRNA-30d promotes tumor invasion and metastasis by targeting Galphai 2 in hepatocellular carcinoma. Hepatology 51: 846-856, 2010.

15. Tsai WC, Hsu PW, Lai TC, Chau GY, Lin CW, Chen CM, Lin CD, Liao YL, Wang JL, Chau YP, et al: MicroRNA-122, a tumor suppressor microRNA that regulates intrahepatic metastasis of hepatocellular carcinoma. Hepatology 49: 1571-1582, 2009.

16. Bai S, Nasser MW, Wang B, Hsu SH, Datta J, Kutay H, Yadav A, Nuovo G, Kumar P and Ghoshal K: MicroRNA-122 inhibits tumorigenic properties of hepatocellular carcinoma cells and sensitizes these cells to sorafenib. J Biol Chem 284: 32015-32027, 2009.

17. Li Y, Tan W, Neo TW, Aung MO, Wasser S, Lim SG and Tan TM: Role of the miR-106b-25 microRNA cluster in hepatocellular carcinoma. Cancer Sci 100: 1234-1242, 2009.

18. Fan MQ, Huang CB, Gu Y, Xiao Y, Sheng JX and Zhong L: Decrease expression of microRNA-20a promotes cancer cell proliferation and predicts poor survival of hepatocellular carcinoma. J Exp Clin Canc Res 32: 21, 2013.

19. Fukamachi $\mathrm{H}$ and Ito $\mathrm{K}$ : Growth regulation of gastric epithelial cells by Runx3. Oncogene 23: 4330-4335, 2004.

20. Peng Z, Wei D, Wang L, Tang H, Zhang J, Le X, Jia Z, Li Q and Xie K: RUNX3 inhibits the expression of vascular endothelial growth factor and reduces the angiogenesis, growth, and metastasis of human gastric cancer. Clin Cancer Res 12: 6386-6394, 2006.

21. Hsu PI, Hsieh HL, Lee J, Lin LF, Chen HC, Lu PJ and Hsiao M Loss of RUNX3 expression correlates with differentiation, nodal metastasis, and poor prognosis of gastric cancer. Ann Surg Oncol 16: 1686-1694, 2009 .

22. Chuang LS and Ito Y: RUNX3 is multifunctional in carcinogenesis of multiple solid tumors. Oncogene 29: 2605-2615, 2010.

23. Subramaniam MM, Chan JY, Soong R, Ito K, Yeoh KG, Wong R, Guenther T, Will O, Chen CL, Kumarasinghe MP, et al: RUNX3 inactivation in colorectal polyps arising through different pathways of colonic carcinogenesis. Am J Gastroenterol 104: 426-436, 2009.
24. Li QL, Ito K, Sakakura C, Fukamachi H, Inoue K, Chi XZ, Lee KY, Nomura S, Lee CW, Han SB, et al: Causal relationship between the loss of RUNX3 expression and gastric cancer. Cell 109: 113-124, 2002.

25. Wada M, Yazumi S, Takaishi S, Hasegawa K, Sawada M, Tanaka H, Ida H, Sakakura C, Ito K, Ito Y, et al: Frequent loss of RUNX3 gene expression in human bile duct and pancreatic cancer cell lines. Oncogene 23: 2401-2407, 2004.

26. Yanada M, Yaoi T, Shimada J, Sakakura C, Nishimura M, Ito K, Terauchi K, Nishiyama K, Itoh K and Fushiki S: Frequent hemizygous deletion at 1p36 and hypermethylation downregulate RUNX3 expression in human lung cancer cell lines. Oncol Rep 14: 817-822, 2005.

27. Nishio M, Sakakura C, Nagata T, Komiyama S, Miyashita A, Hamada T, Kuryu Y, Ikoma H, Kubota T, Kimura A, et al: RUNX3 promoter methylation in colorectal cancer: Its relationship with microsatellite instability and its suitability as a novel serum tumor marker. Anticancer Res 30: 2673-2682, 2010.

28. Zheng Y, Zhang Y, Huang X and Chen L: Analysis of the RUNX3 gene methylation in serum DNA from esophagus squamous cell carcinoma, gastric and colorectal adenocarcinoma patients. Hepatogastroenterology 58: 2007-2011, 2011.

29. Kitago M, Martinez SR, Nakamura T, Sim MS and Hoon DS: Regulation of $R U N X 3$ tumor suppressor gene expression in cutaneous melanoma. Clin Cancer Res 15: 2988-2994, 2009.

30. Lai KW, Koh KX, Loh M, Tada K, Subramaniam MM, Lim XY, Vaithilingam A, Salto-Tellez M, Iacopetta B, Ito Y, et al; Singapore Gastric Cancer Consortium: MicroRNA-130b regulates the tumour suppressor RUNX3 in gastric cancer. Eur J Cancer 46: 1456-1463, 2010.

31. Liu Z, Chen L, Zhang X, Xu X, Xing H, Zhang Y, Li W, Yu H, Zeng $J$ and Jia J: RUNX3 regulates vimentin expression via miR-30a during epithelial-mesenchymal transition in gastric cancer cells. J Cell Mol Med 18: 610-623, 2014.

32. Nakanishi Y, Shiraha H, Nishina S, Tanaka S, Matsubara M, Horiguchi S, Iwamuro M, Takaoka N, Uemura M, Kuwaki K, et al: Loss of runt-related transcription factor 3 expression leads hepatocellular carcinoma cells to escape apoptosis. BMC Cancer 11: 3, 2011.

33. Ladeiro Y, Couchy G, Balabaud C, Bioulac-Sage P, Pelletier L, Rebouissou S and Zucman-Rossi J: MicroRNA profiling in hepatocellular tumors is associated with clinical features and oncogene/tumor suppressor gene mutations. Hepatology 47: 1955-1963, 2008

34. Connolly E, Melegari M, Landgraf P, Tchaikovskaya $\mathrm{T}$, Tennant BC, Slagle BL, Rogler LE, Zavolan M, Tuschl T and Rogler CE: Elevated expression of the miR-17-92 polycistron and miR-21 in hepadnavirus-associated hepatocellular carcinoma contributes to the malignant phenotype. Am J Pathol 173: $856-864,2008$

35. Varnholt H, Drebber U, Schulze F, Wedemeyer I, Schirmacher P, Dienes HP and Odenthal M: MicroRNA gene expression profile of hepatitis $\mathrm{C}$ virus-associated hepatocellular carcinoma. Hepatology 47: 1223-1232, 2008.

36. Lee JH,Pyon JK, Kim DW, Lee SH, Nam HS, Kang SG, Kim CH, Lee YJ, Chun JS and Cho MK: Expression of RUNX3 in skin cancers. Clin Exp Dermatol 36: 769-774, 2011.

37. Lee CWL, Chuang LSH, Kimura S, Lai SK, Ong CW, Yan B, Salto-Tellez M, Choolani M and Ito Y: RUNX3 functions as an oncogene in ovarian cancer. Gynecol Oncol 122: 410-417, 2011.

38. Lin FC, Liu YP, Lai CH, Shan YS, Cheng HC, Hsu PI, Lee CH, Lee YC, Wang HY, Wang CH, et al: RUNX3-mediated transcriptional inhibition of Akt suppresses tumorigenesis of human gastric cancer cells. Oncogene 31: 4302-4316, 2012.

39. Voon DC, Wang H, Koo JK, Nguyen TA, Hor YT, Chu YS, Ito K, Fukamachi H, Chan SL, Thiery JP, et al: Runx3 protects gastric epithelial cells against epithelial-mesenchymal transition-induced cellular plasticity and tumorigenicity. Stem Cells 30: 2088-2099, 2012.

40. Tanaka S, Shiraha H, Nakanishi Y, Nishina S, Matsubara M, Horiguchi S, Takaoka N, Iwamuro M, Kataoka J, Kuwaki K, et al: Runt-related transcription factor 3 reverses epithelialmesenchymal transition in hepatocellular carcinoma. Int $\mathbf{J}$ Cancer 131: 2537-2546, 2012.

41. Zhang Y, Zheng L, Ding Y, Li Q, Wang R, Liu T, Sun Q, Yang H, Peng S, Wang W, et al: MiR-2Oa induces cell radioresistance by activating the PTEN/PI3K/Akt signaling pathway in hepatocellular carcinoma. Int J Radiat Oncol Biol Phys 92: 1132-1140, 2015. 